\title{
A New Twist in the Abdominal Aortic Aneurysm Story
}

\author{
Martin David Tilson, MD ${ }^{1}$ \\ ${ }^{1}$ Department of Surgery, St. Luke's/Roosevelt Hospital Center, \\ College of Physicians and Surgeons, Columbia University Irving \\ Medical Center, New York, New York
}

Aorta (Stamford) 2021;9:167-168.
Address for correspondence Martin David Tilson, MD, Department of Surgery, St. Luke's/Roosevelt Hospital Center, College of Physicians and Surgeons, Columbia University Irving Medical Center, New York, NY (e-mail: mdt1@columbia.edu).
Forty years ago, I published a paper that challenged the dogma that the abdominal aortic aneurysm (AAA) was caused by atherosclerosis. This conclusion was based on a comparison of clinical features of 50 patients with AAA and 50 patients with arterial occlusive disease (AOD). An unexpected finding was that men with AAA outnumbered women by a ratio of $7: 1$, while there were more women than men in the AOD group. ${ }^{1}$

This remarkable discrepancy in incidence by sex had several possible explanations. First, we considered that the AAA gene might be sex linked on the $\mathrm{X}$ chromosome. There was precedent for this in the spontaneously aneurysm-prone blotchy mouse, which has a point mutation in the TIMP-1 gene on the $\mathrm{X}$ chromosome. Only the affected males get aneurysms. ${ }^{2}$

So, we sequenced the TIMP-1 gene in a human subject, and we found a single-nucleotide polymorphism (SNP). ${ }^{3}$ This finding was confirmed by others, but it turned out to be trivial because the SNP was in the third position of the codon. Until recently, there has been no convincing explanation for male predominance.

In 2019, Tang et $\mathrm{al}^{4}$ reported that there is significant loss of the Y chromosome (LOY) in male patients with AAA. Although LOY occurs in males during aging, the degree of LOY in AAA patients was greater than expected by comparison to age-matched normal and AOD controls.

If LOY is important in males, the same might explain the occurrence of AAA in Turner syndrome (XO). It is interesting that there is a growing list of LOY-associated diseases. Several of these involve inflammation and autoimmunity. Examples include Hashimoto's thyroiditis ${ }^{5}$ and rheumatoid arthritis. ${ }^{6}$ For a comprehensive discussion, see a recent paper by Guo et al. ${ }^{7}$

Our laboratory had also described, in the early 1990's, roles for inflammation in $\mathrm{AAA}^{8}{ }^{8}$ as well as autoimmunity, ${ }^{9}$ and destruction of aortic integrity by matrix metalloproteinases. ${ }^{10}$ Investigation of these aspects of AAA pathobiology has led to the publication of approximately 19,000 scientific papers, according to recent searches of Google Scholar.

LOY has also been associated with the occurrence of numerous cancers and disorders of blood and bone marrow. We noticed many years ago that malignancies were not unusual in our AAA patients. The State of Connecticut had an excellent tumor registry, so one of our coinvestigators undertook the effort to search our lists of AAA and AOD patients. A difference in favor of an association of AAA with malignancy was observed. ${ }^{11}$ However, the numbers were small, and the statistical significance was unimpressive $(p<0.05)$. The finding was not confirmed initially but the issue should be revisited. ${ }^{12}$

Finally, the present situation allows me to reflect on a philosophical chestnut. In 2006, a New York Academy of Sciences Symposium on the AAA was organized by myself, Helena Kuivaniemi, and Gilbert R. Upchurch, Jr. The last two talks of the meeting were given by Dr. Kuivaniemi and me. We represented the two alternative approaches to discover the AAA susceptibility gene (or genes). Dr. Kuivaniemi discussed genome wide screening (GWS), ${ }^{13}$ and I spoke for the candidate gene approach (CGA). ${ }^{14}$ The major advantage of GWS is that it is highly objective because it proceeds with zero assumptions about where in the genome to go looking. I call it the "brute force" approach. The disadvantage is that it is highly resource intensive, requires a great many patients, and involves many cooperating investigators and institutions, not to mention that it is expensive. On the other hand, the CGA can be undertaken by one or a few investigators with a small laboratory and limited resources. All that is needed is an in-depth knowledge of the pathobiology of the disease. It is David versus Goliath. In the case of AAA, it appears that the little guys may have won this time, pending confirmations. published online October 29, 2021
DOI https://doi.org/ $10.1055 / \mathrm{s}-0041-1729917$. ISSN 2325-4637.
(C) 2021. The Author(s).

This is an open access article published by Thieme under the terms of the Creative Commons Attribution License, permitting unrestricted use, distribution, and reproduction so long as the original work is properly cited. (https://creativecommons.org/licenses/by/4.0/)

Thieme Medical Publishers, Inc., 333 Seventh Avenue, 18th Floor, New York, NY 10001, USA 


\section{Funding}

None.

\section{Conflict of Interest}

The authors declare no conflict of interest related to this article.

\section{Acknowledgments}

None.

\section{References}

1 Tilson MD, Stansel HC. Differences in results for aneurysm vs occlusive disease after bifurcation grafts: results of 100 elective grafts. Arch Surg 1980;115(10):1173-1175

2 Brophy CM, Tilson JE, Braverman IM, Tilson MD. Age of onset, pattern of distribution, and histology of aneurysm development in a genetically predisposed mouse model. J Vasc Surg 1988;8 (01):45-48

3 Tilson MD, Reilly JM, Brophy CM, Webster EL, Barnett TR. Expression and sequence of the gene for tissue inhibitor of metalloproteinases in patients with abdominal aortic aneurysms. J Vasc Surg 1993;18(02):266-270

4 Tang D, Han Y, Lun Y, et al. Y chromosome loss is associated with age-related male patients with abdominal aortic aneurysms. Clin Interv Aging 2019;14:1227-1241
5 Persani L, Bonomi M, Lleo A, et al. Increased loss of the Y chromosome in peripheral blood cells in male patients with autoimmune thyroiditis. J Autoimmun 2012;38(2,3):J193-J196

6 Miyashita Y, Ono M, Ono M, Ueki H, Kurasawa K. Y chromosome microchimerism in rheumatic autoimmune disease. Ann Rheum Dis 2000;59(08):655-656

7 Guo X, Dai X, Zhou T, et al. Mosaic loss of human Y chromosome: what, how and why. Hum Genet 2020;139(04):421-446

8 Brophy CM, Reilly JM, Smith GJ, Tilson MD. The role of inflammation in nonspecific abdominal aortic aneurysm disease. Ann Vasc Surg 1991;5(03):229-233

9 Gregory AK, Yin NX, Capella J, Xia S, Newman KM, Tilson MD. Features of autoimmunity in the abdominal aortic aneurysm. Arch Surg 1996;131(01):85-88

10 Newman KM, Jean-Claude J, Li H, et al. Cellular localization of matrix metalloproteinases in the abdominal aortic aneurysm wall. J Vasc Surg 1994;20(05):814-820

11 Tilson MD, Fieg EL, Harvey M. Malignant neoplasia in patients with abdominal aortic aneurysms. Arch Surg 1984;119(07):792-794

12 Chan EL, Belem P, Ciocca RG, et al. Incidence of cancer and abdominal aortic aneurysms. A logistic regression analysis. Ann N Y Acad Sci 1996;800:68-73

13 Kuivaniemi H, Kyo Y, Lenk G, Tromp G. Genome-wide approach to finding abdominal aortic aneurysm susceptibility genes in humans. Ann N Y Acad Sci 2006;1085:270-281

14 Tilson MDIII,RoCY.The candidate gene approach to susceptibility for abdominal aortic aneurysm: TIMP1, HLA-DR-15, ferritin light chain, and collagen XI-Alpha-1. Ann N Y Acad Sci 2006;1085:282-290 